\title{
Variaciones en las conductas no coitales de las mujeres
}

\section{Variations of noncoital behavior of famales}

\author{
Encarnación Sueiro, Antonio López-Castedo, José Luís Diéguez
}

Fac. de CC. de la Educación. (Univ. de Vigo-Campus de Ourense)

\section{Resumen}

En esta investigación se analizan los cambios ocurridos en las últimas conductas no coitales, en las mujeres, durante un intervalo de 10 años, mediante un estudio longitudinal, diacrónico o transversal y de tendencia sobre una muestra de 3309 (curso 97-98) y 1408 (07-08) chicas universitarias, que realizaban el primer y último curso en la Universidad de Vigo. Se les aplicó una encuesta estructurada, anónima y voluntaria en la que se recogía la edad y variables sexuales no coitales (autoestimulación, edad de inicio y frecuencia, existencia de actividad sexual con otra persona y existencia de diferenctes conductas no coitales y el coito, así como su edad de comienzo). Los datos se analizan mediante el SPSS 21.0, obteniéndose Media y Desviación Típica para las variables cuantitativas y Frecuencias y Porcentajes para las cualitativas. La edad media de las universitarias del curso 97-98 es de 20.61 años y la del curso 07-08, de 20.96 años.

Palabras clave: Evolución, conductas no coitales, universitarias

\begin{abstract}
In the last decades, sexuality is one of the facets that raises more interest among evolutionary researchers. In this research the change occurred in the noncoital behavior that are analyzed in young women, for a period of 10 years. It took a very lengthy study, diachronic o transversal, with a trend over a sample of 3309 woman university students (course 97-98) and 1408 (course 07-08), in they were in their first and last year of their university course, in the University of Vigo. It was applied a structured survey, anonymous and voluntary, in which was the ages and variables of noncoital behavior. The average age of university females, course 97-98, was of the ages 20.61 years old and the course $07-08$, was of the ages 20.96 years old. The data was anaylzed using the statistical program SPSS 21.0.
\end{abstract}

Keywords: evolution, noncoital behaviors, female university students.

\section{Objetivo}

Conocer la evolución de las conducta sexual no coitales de las mujeres.

\section{Método}

Es un estudio longitudinal, diacrónico o transversal y de tendencia, desarrollado sobre una muestra de 3223 y de 1362 jóvenes universitarias que realizaban el primer y último curso, en el Campus de Vigo, de Ourense y de Pontevedra, de la Universidad Sur de Galicia, en el curso 1997-1998 y 2007-2008, respectivamente.

A toda se le aplicó una encuesta estructurada, anónima y voluntaria en la que se recogía la edad y variables referidas a las relaciones sexuales no coitales: existencia de autoestimulación, edad de inicio y frecuencia, actividad sexual realizada con otra persona diferente del coito, diferentes conductas sexuales ejecutadas (cita, beso, beso profundo, estimulación de los senos por encima de la ropa, estimulación de los senos debajo de la ropa, estimulación genital activa, estimulación genital pasiva, contacto intergenital, coito vaginal/anal con una o varias parejas) y su edad de comienzo.

Los datos se analizan mediante el programa estadístico SPSS 17.0. para hallar la Media y Desviación Típica (S), para la descripción de las variables cuantitativas, y la frecuencia y porcentaje, para la descripción de las variables cualitativas. Para hallar las diferencias significativas, empleamos la prueba t, y la Chi cuadrado.

En esta investigación se analizan los cambios ocurridos en las últimas conductas no coitales, en las mujeres, durante un intervalo de 10 años, mediante un estudio longitudinal, diacrónico o transversal y de tendencia sobre una muestra de 3309 (curso 97-98) y 1408 (07-08) chicas universitarias, que realizaban el primer y último curso en la Universidad de Vigo. Se les aplicó una encuesta estructurada, anónima y voluntaria en la que se recogía la edad y variables sexuales no coitales (autoestimulación, edad de inicio y frecuencia, existencia de actividad sexual con otra persona y existencia de diferenctes conductas no coitales y el coito, así como su edad de comienzo). Los datos se analizan mediante el SPSS 21.0, obteniéndose Media y Desviación Típica para las variables cuantitativas y Frecuencias y Porcentajes para las cualitativas.

La edad media de las universitarias del curso 97-98 es de 20.61 años y la del curso 07-08, de 20.96 años. 


\section{Resultados}

Las mujeres que se autoestimularon, suponen el $52.0 \%$, del curso $97-98$, y el $62.8 \%$, del curso $07-08$. Por el contrario, las mujeres que afirmaron no haberse masturbado son un $44.0 \%$ y un $32.7 \%$, respectivamente, encontrándose diferencias significativas entre ambos $\operatorname{cursos}\left(\chi^{2}=68.56 ; p=0.000\right)$.

Sobre la frecuencia de la autoestimulación el porcentaje más elevado $(36.0 \%)$ de mujeres que se masturbaron en el curso 97-98 se encuentra en la frecuencia de menos de una vez al mes, decreciendo a un $33.6 \%$ en el curso $07-08$. El $30.1 \%$ y el $31.6 \%$, de la primera y segunda muestra respectivamente, afirmaron haberse masturbado de 1 a 3 veces al mes. Por otro lado, el $14.6 \%$ de las mujeres del curso $97-98$ y el $11.8 \%$ de las del curso 07-08 aseguraron haberse masturbado sólo la primera vez. A su vez, es de destacar que un $3.4 \%$ en la primera muestra y un $6.6 \%$ de la segunda manifestaron haberse autoestimulado 4 ó más veces por semana y de 1 a 3 veces a la semana se ha masturbado el $13 \%$ de las mujeres de cada grupo. Se ponen de manifiesto diferencias significativas entre las mujeres de ambos grupos $\left(\chi^{2}=18.35 ; \mathrm{p}=0.003\right)$.

La edad en que las mujeres se iniciaron en la conducta autoestimulatoria es el más elevado, se manifiesta en la franja de edad de 13 a 16 años, siendo de un $41.2 \%$ para las mujeres del curso 97-98, e incrementándose a un $46.9 \%$ en las mujeres del curso $07-08$. Por otra parte, el $20.1 \%$ de la primera muestra y el $15.8 \%$ de la segunda muestra comenzaron antes de los 12 años, mientras el $38.1 \%$ y $35.8 \%$, respectivamente, tenía 17 o más años, encontrándose diferencias significativas entre las mujeres de ambos cursos $\left(\chi^{2}=11.31 ; \mathrm{p}=0.023\right)$.

En cuanto a sí las mujeres realizaron algún tipo de actividad sexual con otra persona, se percibe que el $78.4 \%$, de la muestra del curso $97-98$ y el $89.2 \%$ de la muestra del curso 07-08, revelaron que sí habían realizado algún tipo de actividad sexual con otra persona, por el contrario, sólo el $6 \%$ de las primeras y el $3.8 \%$ de las segundas, no habían iniciado alguna actividad sexual. La diferencia es significativa entre ambos grupos de mujeres $\left(\chi^{2}=71.17 ; p=0.000\right)$.

En relación a las diferentes conductas realizadas por las mujeres, el porcentaje de mujeres, del curso 97-98 que declaran que tuvieron cita es de $72.8 \%$, ascendiendo, en el curso 07-08, a un 84.0\%. Los porcentajes en esta variable se incrementan en las diferentes conductas sexuales: beso (del $77.7 \%$ sube a un $87.3 \%$ ), beso profundo (del $81.3 \%$ pasa a un $86.0 \%$ ), estimulación de los senos por encima de la ropa (del $67.0 \%$ sube a un $77.3 \%$ ), estimulación de los senos debajo de la ropa (del $66.5 \%$ asciende a un $78.0 \%$ ), estimulación genital activa (del $54.2 \%$ sube a un $74.8 \%$ ), estimulación genital pasiva (del $50.9 \%$ pasa a un $71.0 \%$ ), contacto intergenital (del $46.1 \%$ se incrementa a un $69.2 \%$ ), coito vaginal/anal con varias parejas (del $2.4 \%$ pasa a $11.0 \%$ ), siendo, por tanto, siempre mayor para la muestra del curso 07-08. Por otra parte, es de destacar el aumento de las mujeres que realizaron coito con una persona, pasando de un $40.9 \%$, en el curso $97-98$, a un $72.5 \%$, en el curso 07-08.

Se ponen de manifiesto diferencias significativas en todas las conductas sexuales mencionadas en las mujeres: $\operatorname{cita}\left(\chi^{2}=58.40 ; \mathrm{p}=0.000\right)$, beso $\left(\chi^{2}=43.52 ; \mathrm{p}\right.$ $=0.000)$, beso profundo $\left(\chi^{2}=14.544 ; \mathrm{p}=0.000\right)$, estimulación de los senos encima de la ropa $\left(\chi^{2}=42.68\right.$; $\mathrm{p}=0.000)$, estimulación de los senos debajo de la ropa $\left(\chi^{2}=61.03 ; \mathrm{p}=0.000\right)$, estimulación genital activa $\left(\chi^{2}=\right.$ $182.49 ; \mathrm{p}=0.000)$, estimulación genital pasiva $\left(\chi^{2}=\right.$ $164.30 ; \mathrm{p}=0.000)$, contacto intergenital $(213.98 ; \mathrm{p}=$ $0.000)$, coito vaginal/anal con una persona $\left(\chi^{2}=366.39\right.$; $\mathrm{p}=0.000)$ y coito vaginal/anal con varias personas $\left(\chi^{2}=\right.$ $153.86 ; \mathrm{p}=0.000)$.

Por lo que respecta a las edades de inicio de las diferentes conductas sexuales se observa que la edad de comienzo de la cita es de 14.64 años para las mujeres del curso 97-98 y de 14.23 años para las del curso 0708. Las edades de inicio en los comportamientos sexuales analizados son mayores para las mujeres del curso 97-98 con respecto a las del curso 07-08 en las siguientes conductas sexuales: beso (del 14.83 años y de 13.88 años, respectivamente), beso profundo (de 15.55 años y de 14.58 años, respectivamente), estimulación de los senos encima de la ropa (de 16.56 años y de 15.83 años, respectivamente), estimulación de los senos debajo de la ropa (de 16.97 años y de 16.25 años, respectivamente), estimulación genital activa (de 17.74 años y de 16.76 años, respectivamente), estimulación genital pasiva (de 17.67 años y de 16.77 años), contacto intergenital (de 18.11 años y de 17.11 años, respectivamente), coito vaginal/anal con varias parejas (18.57 años y 17.81 años, respectivamente). Cabe destacar la menor edad de comienzo coital de las mujeres del curso 07-08 (17.41 años) si la comparamos con la de las mujeres del curso 97-98 (18.42 años). Las diferencias son significativas entre las mujeres de ambos grupos en las edades de inicio en las siguientes prácticas sexuales: beso $(\mathrm{t}=5.99 ; \mathrm{p}=0.014)$, estimulación de los senos encima de la ropa $(\mathrm{t}=7.59 ; \mathrm{p}$ $=0.006)$, estimulación genital activa $(\mathrm{t}=5.104 ; \mathrm{p}=$ $0.024)$ y pasiva $(\mathrm{t}=4.24 ; \mathrm{p}=0.040)$, sin embargo no se muestran diferencias significativas en las conductas sexuales de: cita $(\mathrm{t}=1.48 ; \mathrm{p}=0.224)$, beso profundo $(\mathrm{t}=$ $0.01 ; p=0.912$ ), estimulación de los senos debajo de la ropa $(\mathrm{t}=0.16 ; \mathrm{p}=0.681)$, contacto intergenital $(\mathrm{t}=1.41$; $\mathrm{p}=0.234)$ y coito vaginal/anal con una pareja y varias parejas $(\mathrm{t}=2.72 ; \mathrm{p}=0.100)$.

\section{Discusión}

En nuestros datos se observan un incremento significativo en las mujeres en la conducta de autoestimulación, debido a que en el curso $1997-98$ se masturbaban un $52.0 \%$ y se incremento a un $62.8 \%$ en el curso 2007-08, esto nos permite afirmar que la mujer actual se masturba en mayor medida que la de hace 10 años. Con población universitaria, se encuentran tasas 
de autoestimulación de un 60.6\% (Malo de Molina, 1988); un 53\% (Sueiro y Diéguez, 2001), porcentaje que coincide con el obtenido en este estudio con las universitarias del curso 1997-98; frente a un 34.2\% que afirma no haberla realizado (Van-der Hofstadt et al., 2007-2008), resultados estos que coinciden con las mujeres del curso 2007-08.

Cuando pasamos a valorar la variable relativa a la frecuencia de la autoestimulación, los porcentajes más altos se encuentran en el intervalo de menos de una vez al mes (36\% de las mujeres del curso $1997-98$ y $33.6 \%$ de la del curso 2007-08) y en el intervalo de 1 a 3 veces al mes $(30.1 \%$ de las mujeres del curso $1997-98$ y $31.6 \%$ de la del curso 2007-08), reiterando que la mujer actual se masturba con mayor frecuencia que la mujer de hace 10 años. Los resultados obtenidos por las mujeres del curso 1997-98, corroboran las investigaciones de Cabello et al. (1993), Diz et al. (2003), López (1990) y Sueiro y Diéguez (2001).

Cuando nos referimos a la edad de inicio de la primera autoestimulación, los porcentajes más altos se encuentran en la franja de edad de 13 a 16 años en las dos muestras (curso 1997-98 y 2007-08), siendo los porcentajes los del $41.2 \%$ y $46.9 \%$, respectivamente; destacando que un $34.0 \%$ de la primera muestra y un $32.2 \%$ de la segunda muestra, comenzaron en la franja de edad de 17 a 20 años, siendo las universitarias del curso 2007-08 las que se iniciaron antes en la práctica de autoestimulación que las del curso 1997-98.

Hay algunos estudios que hacen referencia al descenso que se produce en la edad de la primera masturbación, en el caso de las chicas. Dreyer (1982), refiere que en los comienzos de la década de los 70 , alrededor del $30 \%$ de las chicas se habían masturbado hacia la edad de los 15 años; mientras que, a finales de la misma década, los porcentajes eran de $45 \%$. Mesa et al. (2004), revelan que las mujeres iniciaron la conducta autoestimulatoria entre los 14-16 años.

Observando la variable relativa a haber tenido alguna actividad sexual con otra persona, encontramos que un $78.4 \%$ de las mujeres, del curso $97-98$, y el $89.2 \%$, del curso 2007-08, manifestaron haberla realizado, poniéndose de manifiesto que, después de 10 años, hay mayor número de mujeres que tuvieron algún tipo de actividad sexual. Así, García et al (1995), sobre universitarios navarros, informan que un $8.1 \%$ de las mujeres han tenido poco o ningún contacto con el sexo opuesto, y un $17.8 \%$ de las mujeres tienen una experiencia limitada de actividades sexuales (besos y caricias por encima de la ropa), porcentajes que concuerdan con los encontrados con la muestra del curso 1997-98. Por su parte, en la $2^{\text {a }}$ Encuesta Schering de Sexualidad y Anticoncepción en la juventud Española (2005), realizada entre jóvenes con edades comprendidas entre 15 y 24 años, se constata que el $87.0 \%$ de las chicas han mantenido relaciones sexuales en alguna ocasión (con o sin penetración). Estos datos coinciden con la Encuesta de Salud y Hábitos Sexuales del INE (2003) y el IJE-2008, aunque el grupo de edad analizado es de 18 a 29 años, indicando que el 86.2\% de las mujeres declaraba haber tenido relaciones sexuales completas e incompletas (Suárez, Belza y De la Fuente, 2006). Estos resultados corroboran los obtenidos con las mujeres del curso 2007-08.

Si nos centramos en las prácticas sexuales realizadas (beso, beso profundo, estimulación senos, estimulación genital, contacto intergenital y coito), hay un incremento significativa cuando pasamos del curso 1997-98 al curso 2007-08, en todas ellas. Cabe destacar el coito vaginal/anal con una persona, donde el porcentaje asciende del $40.9 \%$ en el curso $1997-98$ al $72.3 \%$ en el curso 2007-08. Estos resultados vienen a corroborar la investigación de Sueiro y Diéguez (2001), donde cabe destacar que a medida que nos aproximamos al coito, la presencia de los comportamientos sexuales tiende a disminuir, el porcentaje desciende del $75.9 \%$, para el beso entorno al $64 \%$ cuando se trata de estimulación de los senos, se reduce a un $43.9 \%$ para el contacto intergenital, y para el coito vaginal/anal al $38.5 \%$.

Por otra parte, si realizamos un análisis cronológico de los diferentes estudios sobre población universitaria en España, observamos cómo el porcentaje de sujetos que realiza el coito se incrementa paulatinamente según pasan los años, estos resultados corroboran los datos encontrados del curso 1997-98 y 2007-08. Lameiras (1997), sobre alumnado de primer curso de la Universidad de Vigo (Campus de Ourense), revela que el $33.3 \%$ de las chicas encuestados ha mantenido relaciones sexuales coitales; Sueiro y Diéguez (2001), realizada en el curso 1997-98, con mujeres de primer curso de la Universidad de Vigo, indican que el 38.5\% de las mismas tuvo coito vaginal/anal con una pareja.

Si nos centramos en los diferentes estudios del IJEs, desde el año 1996 hasta la actualidad, también reafirman nuestros datos, ya que en 12 años se observa que las relaciones coitales en las mujeres de 18 a 20 años pasan de un $35 \%$ a un $74.1 \%$, ambos porcentajes están en la misma línea que los encontrados en los cursos 1997-98 y 2007-08. (IJE-96: un 52\% afirma que ha tenido relaciones sexuales completas para las mujeres de edades comprendidas entre los 15 a 29 años, para los tramos de edad de 18 a 20 años sería de un $35 \%$ y para el tramo de edad comprendido entre 21 a 24 años de un 64\%; IJE-2000: un 53\% de las mujeres de 15 a 29 años, para el tramo de edad de 18 a 20 años sería un $42 \%$ y para el tramo de edad de 21 a 24 años de 54\%; IJE-2004: un $79.1 \%$ de las mujeres de 15 a 29 tuvieron coito, por tramos de edad sería un $72.9 \%$ de las mujeres de 18 a 20 años y el $89.3 \%$ de las mujeres de 21 a 24 años; IJE-2008: un $81.8 \%$ de las mujeres tuvieron coito, por tramos de edad sería un $74.1 \%$ de las mujeres de 18 a 20 años y de un $94.4 \%$ de 21 a 24 años.

Cuando analizamos muestras de alumnado de enseñanza no universitaria, Diz et al. (2003), sobre 
población del sur de Galicia, refieren que el $29.4 \%$ de las mujeres tuvieron su primer coito; Lameiras et al. (2004b), sobre alumnado orensano, afirman que un 27\% de las chicas son sexualmente activas; Mesa et al. (2004), indican que un $22.5 \%$ de las mujeres practicaron coito; y, Teva et al. (2009), manifiestan que el $29.9 \%$ tenían experiencia sexual coital.

A la hora de evaluar la variable referida a la edad de inicio de las diferentes prácticas sexuales realizadas, encontramos un descenso en la misma; es decir, las mujeres del curso 2007-08 comenzaron a edades más tempranas que las mujeres del curso 1997-98, en algunas de las prácticas estudiadas este descenso es significativo (beso, estimulación de los senos encima de la ropa, estimulación genital activa y pasiva). Por lo que hace referencia al coito, la edad media descendió desde los 18.4 años para las mujeres del curso 1997-98 a 17.4 años para las mujeres del curso 2007-08.

Atendiendo a las prácticas no coitales, en la investigación de Oraá (1996), sobre jóvenes riojanos, manifestaba que las primeras prácticas a las que se acceden las mujeres son los besos, aproximadamente a los 14 años, esta edad coincide con las mujeres del curso 1997-98. Por su parte, Mesa et al. (2004), señalan que el $60.7 \%$ tuvieron su primer beso a los 14-16 años y el $92.2 \%$ a los 16 años, resultados que no son totalmente equiparables con la muestra del curso 2007-08, debido a que esta investigación se centra más en la edad media y este estudio se utilizan diferentes tramos de edad.

A su vez, la encuesta del INE-2003, sitúa la edad de inicio de los coitos en las mujeres sobre los 18.2 años, porcentaje que se asemeja al encontrado en esta investigación; sin embargo, se debe tener en cuenta que en este trabajo, una es anterior y otra es posterior, y la tendencia es a ir disminuyendo la edad de inicio de las relaciones coitales con el paso del tiempo. Así mismo, los resultados de la $2^{\text {a }}$ Encuesta Schering de Sexualidad y Anticoncepción en la Juventud Española (2005), establecen que la edad media de las chicas es de 16.9 años, pero para las jóvenes entre 20 y 24 años, las edades se incrementan a 17.3 años, edades que coinciden totalmente con las halladas en la muestra del curso 07-08. En el estudio AFRODITA (Bosch et al., $2009)$, se observa que la gran mayoría (31.7\%) tuvo su primer coito entre los 19 y 21 años, el $7.2 \%$ lo inició antes de los 16 años y un $15.9 \%$ lo refiere después de los 25 años; sin embargo, si se tiene en cuenta sólo las mujeres entre 18 y 25 años, los porcentajes cambian y el grupo de edad en que la mayoría (45.9\%) tuvo su primer coito pasa a ser de $17-18$ años y el $16.7 \%$ de las mismas lo tuvieron a los 16 años o antes. En este mismo estudio se observa una tendencia en las mujeres más jóvenes a iniciar las relaciones coitales más tempranamente que las mujeres de edades más avanzadas. Estos resultados están en la misma línea que los encontrados con las mujeres del curso 2007-08. En el estudio de Ramírez et al. (2002), sobre métodos anticonceptivos en la población Andaluza, con edades comprendida entre los 15 y 49 años, la edad media de inicio de las relaciones coitales en la mujeres es de 18.6 años, resultados que concuerdan con los hallados en la muestra del curso 1997-98. La investigación de Lasheras et al. (2005), recalcan que las mujeres inician su primera relación coital después de los 17 años. Por último, Lameiras et al. (2007-08), afirman que la edad de inicio de la actividad coital de la mujeres del Campus de Ourense es de 17.6 años, porcentajes que están en la misma línea de la muestra de mujeres del curso 2007-08. Sin embargo, en el estudio de Amorín y Pulgar (2009), la edad media de las relaciones coitales es de 17.9 años, destacando que la muestra esta formada por mujeres de edades muy variadas (la más joven tiene 15 años y la más mayor 51 años), lo que hace incrementar la media de inicio de las relaciones coitales, en este mismo estudio se señala que las mujeres con mayor edad, han comenzado más tarde a tener relaciones de coito, es decir, se aprecia una tendencia en las mujeres más jóvenes a comenzar antes el coito.

Cuando se baja la edad de las personas investigadas, se observa que las edades de inicio coital también disminuye, así lo ponen de manifiesto las investigaciones de Oraá (1996), revelando que la edad media para practicar el coito en las mujeres fueron los 17 años; Hidalgo et al. (2000), que sitúan la edad del primer coito en la mujer en 16.1 años; Diz et al. (2003), refieren que la edad media del coito es de 17.2 años; Moreno Rodríguez et al. (2004), sitúan la edad en 15.4 años; y Teva et al. (2009b), que reseñan la edad de inicio de las relaciones coitales en la mujer en los 15.0 años.

\section{Referencias}

Amorín, C. y Pulgar, M. (2009). Relación entre coitoedad-parejas en mujeres de las comarcas de $\mathrm{O}$ Carballiño-Ribadavia. Boletín Oficial Sociedade Galega de Contracepción, (15), 43.

Barbosa, R.G., García, F.C., Manzato, A.J., Martins, R.A., y Vieira, F.T. (2006). Conhecimiento sobre DST/AIDS, Hepatites e Conduta Sexual de Universitários de Sao José do Rio Prieto, SP. DST. Jornal brasileiro de Doenças Sexualmente Transmissíveis, 18 (4), 224-230.

Bosch, F.X., Lorincz, A., Muñoz, N.C., Meijer, J.L., y Shah, KV. (2002). The causal relation between human papillomavirus and cervical cancer. Journal of Clinical Pathology, (55), 244-65.

Cabello, F. (1993). Campaña de información sexual para jóvenes. Málaga: Sociedad Malagueña de Sexología. Ayuntamiento de Málaga. Delegación de juventud.

Diz, M.C., Sueiro, E., Chas, M.D., y Diéguez, J.L. (2003). Comportamientos sexuales de adolescentes del medio rural gallego ( $3^{\text {a }}$ Parte). Cuadernos de Medicina Psicosomatica y Psiquiatría de Enlace, (67/68), 46-60. 
Dreyer, P.H. (1982). Sexuality during adolescence. En B.B.Wolman (Ed.), Handbook of developmental psychology (pp. 559-601). New Jersey: Pretice-Hall.

García, J.L., Avis, M., Cobos, F., Biurrum, A.C., Eslava, J.L., Rodrigo, C., Padilla, B., y Tinajas, M.R. (1995). Conductas sexuales de riesgos en universitariosas: Un estudio en la Universidad Pública de Navarra. Cuadernos de Medicina Psicosomática y Psiquiatría de Enlace, (36), 48-60.

INE (2003). Encuesta de Salud y Hábitos Sexuales. Madrid: Instituto Nacional de Estadística.

Injuve, (1996). Informe Juventud en España (1996).

Madrid: Ministerio de Trabajo y Asuntos Sociales.

Injuve, (2000). Informe de la Juventud en España 2000. Madrid: Ministerio de Trabajo y Asuntos Sociales.

Injuve. (2004). Condiciones de vida y situación de los jóvenes en España. Informe de juventud en España 2004. Madrid: Ministerio de Trabajo y Asuntos Sociales.

Injuve, (2008). Informe Juventud en España (2008).

Madrid: Ministerio de Trabajo y Asuntos Sociales.

Lameiras, M. y Failde, J.M. (1997). Sexualidad y salud en jóvenes universitarios: actitud, actividad sexual y percepción de riesgo de la transmisión heterosexual del VIH. Análisis y Modificación de Conducta, 23 (93), 28-63.

Lameiras, M., Castro, Y., Calado, M., y González, M. (2004b). Determinantes del inicio de las relaciones sexuales en adolescentes españoles. Cuadernos de Medicina Psicosomática y Psiquiatría de Enlace, (7172), 67-75

Lameiras, M., Nuñez, A.M., Carrera, M.V., y Rodríguez, Y. (2007/2008). Conducta sexual y uso del preservativo masculino en muestra de jóvenes universitarios Gallegos. Cuadernos de Medicina Psicosomática y Psiquiatría de Enlace, (84-85), 49-56.

Lasheras, M.G., Cuñe, J., Bautista J., y Farré J.M. (2005). Hábitos sexuales en jóvenes universitarios. Cuadernos de Medicina Psicosomática y Psiquiatría de Enlace, (74), 57-63.

Malo de Molina, C., Valls, J.M., y Pérez G. (1988). La conducta sexual de los españoles. Barcelona: Ediciones B.

Mesa, M.I., Barella, J.L., y Cobeña, M. (2004). Comportamientos sexuales y uso de preservativos en adolescentes de nuestro entorno. Atención Primaria, 33 (7), 374-380.

Moreno, M.C., Muñoz, M.V., Pérez, P.J., y Sánchez, I. (2004). Los adolescentes españoles y su salud. Un análisis en chicos y chicas de 11 a 17 años. Madrid: Ministerio de Sanidad y Consumo.

Oraá, R. (1996). Hábitos sexuales y conducta preventiva de los/as jóvenes de la Rioja. Cuadernos de Medicina Psicosomática y Psquiatria de Enlace, (40), 51-61.
Ramírez, Y., García, J., De las casas, I., García, E., y Godines, J.A. (2002). Encuesta sobre uso de métodos anticonceptivos en Andalucía. Revista Iberoamericana de Fertilidad y Reproducción Humanas, (VI Congreso SEC), 138-139.

Segunda Encuesta Schering Sexualidad y Anticoncepción en la Juventud Española (2005). Recuperado el 23 de Enero de 2008, de http://www.equipodaphane.es.

Suárez, M., Belza, M.J., y De la Fuente, L. (2006). Encuesta de salud y hábitos sexuales, 2003. Madrid: INE.

Sueiro, E. y Diéguez J.L. (2001). Juventud y sexualidad. Actas do VI Congreso Galaico-Português de Psicopedagogía. Braga (pp. 257-269).

Teva, I., Bermúdez, M.P., y Buela-Casal, G. (2009b). Conductas de riesgo para la infección por el VIH y las enfermedades de transmisión sexual (ETS) en adolescentes en el año 2007: diferencias en función de variables sociodemográficas. Revista Española de Salud Pública, (83), 309-320.

Van-der Hofstadt, C.J., Antón-Ruiz, F.A., Tirado, S., y Navarro-Cremades, F. (2007-2008). Hábitos sexuales en mujeres estudiantes de medicina. Cuadernos de Medicina Psicosomática y Psiquiatría de Enlace, (84/85), 42-45 